\title{
Improving the efficiency of antennas of MIMO communication systems
}

\author{
Victor Obukhovets ${ }^{*}$ \\ Institute of Radio Engineering Systems and Control, Southern Federal University, Rostov-on-Don, \\ Russia
}

\begin{abstract}
The last two decades were marked by increasing of interest in problems of application of the MIMO systems in a radar and wireless communication. Use of the principles of creation of MIMO systems in communication complexes allows increasing the capacity of channels and speed of information transfer due to increase in number of the transferring and reception antennas. In the report questions of modernization of antennas of the wearable mobile communication devices allowing to reduce correlation of channels and to increase spectral system effectiveness of communication are considered. Results of a research of mutual correlation reduction between two antennas of MIMO system are given. Using method of numerical modelling influence of several options of the decoupling structures on interconnection size between radiators and on their directional patterns were investigated. Comparative results of a pilot study of the model of antennas with the outcome device having the best characteristics and without the decoupling device are presented.
\end{abstract}

\section{Introduction}

Modern communication systems are characterized by the continuous growth of the traffic data. So, according to the forecast, in 2021 the volume of traffic has to increase more, than by 200 times in comparison with 2010. Such growth can be provided due to application of MIMO systems principles. Using of them due to increasing the number of communication channels it is possible to achieved high system productivity without expansion of a frequency range of the transmitted signals. Such effect can be characterized by spectral efficiency. Theoretically the last has to increase in proportion to number of communication channels. However implementation of similar communication systems encounters a number of the restrictions which do not allow to reach theoretically predictable benefit because of several reasons.

Today relevant is a finding solutions on increase in spectral efficiency of telecommunication systems. The last determines capacity in a single strip of frequencies and is the most important characteristic of a MIMO system [1 - 4].

Consequently increasing of spectral efficiency of telecommunication systems nowadays became very relevant problem. According the definition the spectral efficiency means the amount of channel capacity in unit frequency band and it directly depends on the mutual

*Corresponding author: vaobuhovec@sfedu.ru 
correlation of channels coefficient. This parameter is caused, mainly, by electromagnetic interaction of antennas in MIMO system [5]. Researches show that at rather big distances between antennas such interaction can be neglected. However for the mobile communication devices which use the MIMO principles such distances are too small, and the mutual coupling between them can cause significant effect on the speed of information transfer and capacity of communication channels.

The aim of this work is modeling and the comparative analysis of methods of mutual coupling suppression between channels for the spectral efficiency increasing.

In wearable mobile communication gadgets microstrip antennas are used most often. Several types of the decoupling methods can be used for reduction of mutual coupling between antennas. By the principle of their operation it is possible to divide them into four groups. The first group methods are based on reduction of an interconnection through external fields [1, 6-7]. Second group methods use the principles of decrease the internal fields amplitudes in a microstrip antennas substrate [2, 8-9, 14]. Third group methods requiere some kind of devices which provide additional mutual coupling using transmission lines, resonators and so on [10-12]. And the fours group use polarization separation for coupling reduction [15-16]. Any combinations of known methods are possible for further decoupling increasing [17]. Some kinds of abovementioned methods are tested in this paper.

The results were obtained using CAD modelling of the antenna with decoupling structure and were performed by means of ANSYS HFSS. All numerical rsults are compared with so called reference antenna [5] which consists of two rectangular patches with coacsial exitation.

\section{Mutual coupling reduction by means of external fields correcting}

There are known a number of antenna constructions which use auxiliary elements above the substrate surface or on it for the aim of mutual coupling reduction. One can try to use some known methods of decoupling of phase arrays radiators for the sake of scan sector increasing [18].

Mutual coupling effects were tested for the three types of decoupling devices (Fig.1). They are: pyramidal metal septum (Fig. 1 a), the strips of the absorber (Fig. 1 b) and EBG $[6,7,21,22]$ structures or "mushroom", which connect patches to the ground using metallic via ( Fig. $1 \mathrm{c}$ ). All of them are situated above the plane of microstrip radiators arrangement.

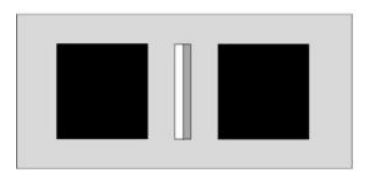

a

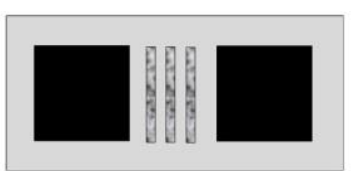

b

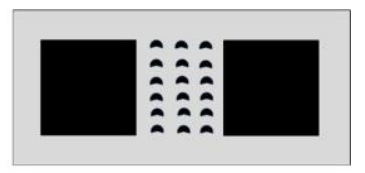

C

Fig. 1. External field correction elements: a - prism, b - absorption strips, c - "mushroom" rods.

Electromagnetic simulations results had shown rather low effectiveness of abovementioned devices for the aim of better decoupling of microstrip antennas. The best results were achieved with metallic prisms parallel patch edges. In the whole frequency band amplitudes of coupling coefficient is about $4.1 \mathrm{~dB}$ smaller then in reference antenna (Fig. 2) and they are below $-20 \mathrm{~dB}$. The devices (Fig. $1 \mathrm{~b}$, c) can change resonant frequency approximately for 1 - 2 percent and can increase beam width slightly. Those 
rather small effects can be improved by increasing the height of elements above the patches plane. Models under consideration (Fig. 1) have elements heights about $5 \mathrm{~mm}$. To make this effects more stronger one should use elements heights which are comparable with wavelength value.

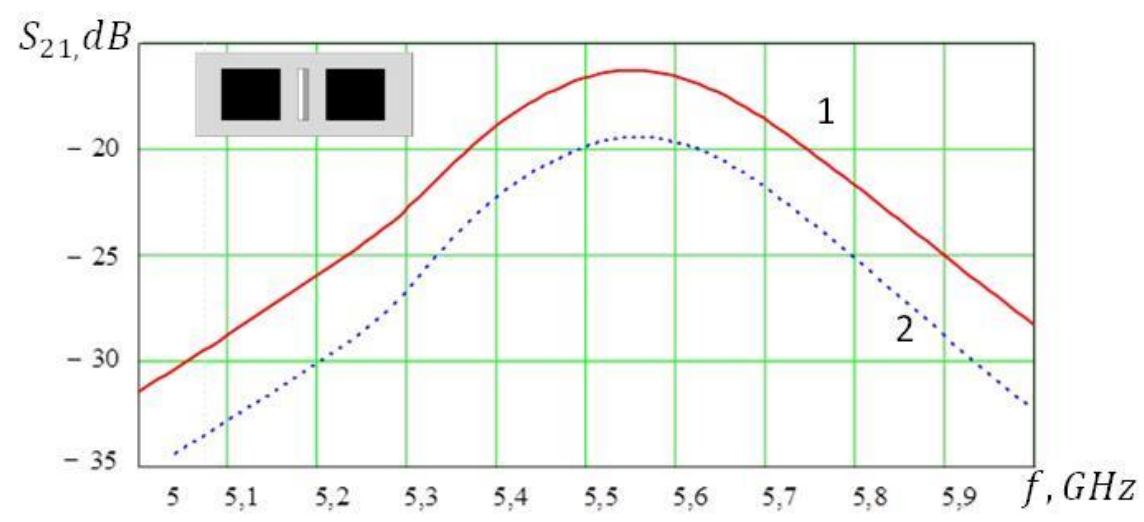

Fig. 2. Frequency dependence mutual coupling coefficient: 1 - reference antenna; 2 -antenna under test

Application of several 3-D metallic walls with height about $5 \mathrm{~mm}$ [18] allows improving the results up to $10-15 \%$.

Rather good characteristics were achieved in [1] by using modified serpentine structures for mutual coupling reduction between two patch antennas.

\section{Application of defected ground plane and dielectric substrate for mutual coupling decrease}

The idea of defected ground structures (DGS) using for improving of MIMO antennas discussed in [19] was modified both for ground plane and dielectric substrate and tested. Three antenna models shown below (Fig. 3) were tested. They have narrow slots in metallic screen of printed circuit board. Compared with reference antenna parallel slots structures improve decoupling for $1 \mathrm{~dB}$ (one slot) and up to $5 \mathrm{~dB}$ (three slots) at the second half of band (three slots). Crossed slots structure does not improve decoupling. All three antennas patterns have a little higher level of back lobes. Highest back lobe level increasing (about 4 percent) has cross slots screen defect. Special researches were made and established that slots width and length almost do not effect on electrical characteristics of antennas except that cases when the length is much more longer than patch.

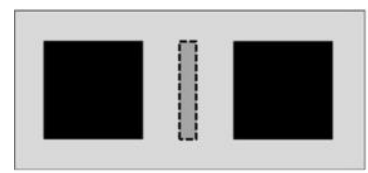

a

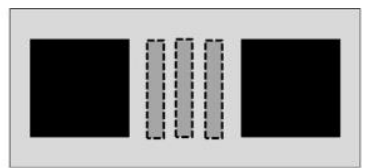

b

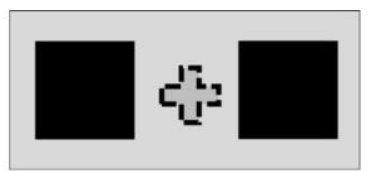

c

Fig. 3. Defected ground plane antennas: a - one slot, b - three slots, c - crossed slots

Frequency dependence of coupling coefficient amount for the antenna with three slots (Fig. 3, b) is shown in Fig. 4 (curve 2). 


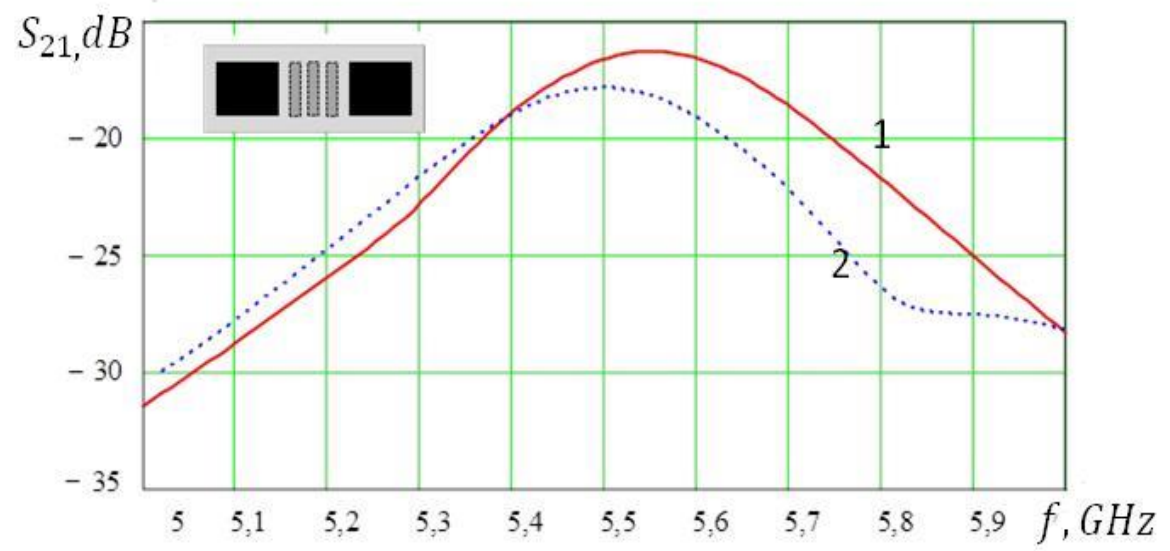

Fig. 4. Three slots in the ground plane: 1 - reference antenna; 2 - antenna with slots.

Internal fields in microstrip antennas could be changed by using "defected" substrates with any inhomogeneities in the dielectric material. One of the simplest of such inhomogeneities are the slots in substrate. Three kinds of slots were investigated (Fig. 5).

Simulation results show that most applicable is the substrate with complicated slot in the form of meander cutout (Fig. 5, c).

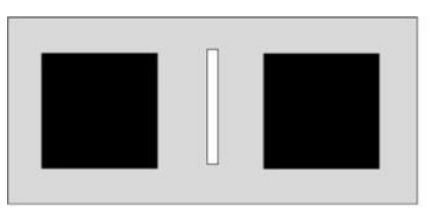

a

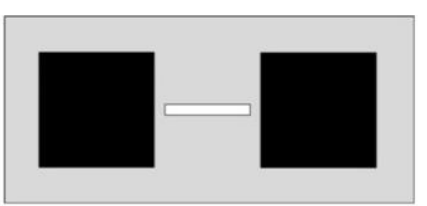

b

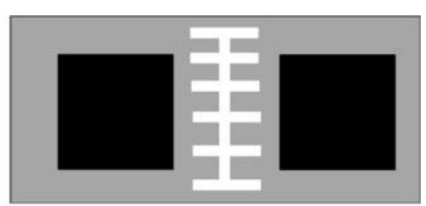

C

Fig. 5. Antennas with defects of dielectric substrate

This type of substrate defect allows to increase the decoupling more than $11 \mathrm{~dB}$ at the requiered frequence (Fig. 6).

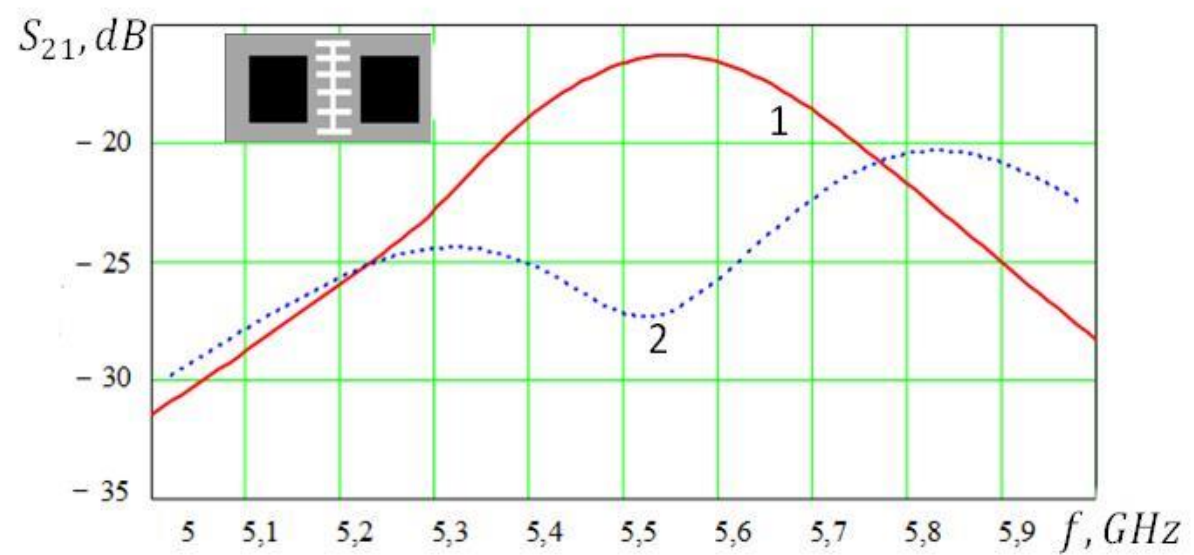

Fig. 6. Decoupling by the cutout in the form of a meander in the dielectric substrate: 1 -reference antenna; 2 - antenna with cutout. 
Such kind of substrate structure results in resonant frequency shift of about 1.5 percent compared with reference antenna. This new substrate does not deteriorate the amount of return losses.

\section{Introduction elements providing additional mutual coupling}

Devices considered in parts 2 and 3 change electromagnetic fields structure in outer or inner area of microstrip MIMO antenna for the sake of mutual coupling reduction. That may be treated as introduction additional electromagnetic coupling which compensate intrinsic coupling between radiators in original antenna.

Along with that it is possible to use any variant of additional galvanic coupling for the same aim $[12,23,24]$. The simplest device is the transmission line connected two patches. More complicate device uses transmission line piece of U-shape [12].

Characteristic of simplest construction with rectangular piece of transmission line is shown on fig. 7 .

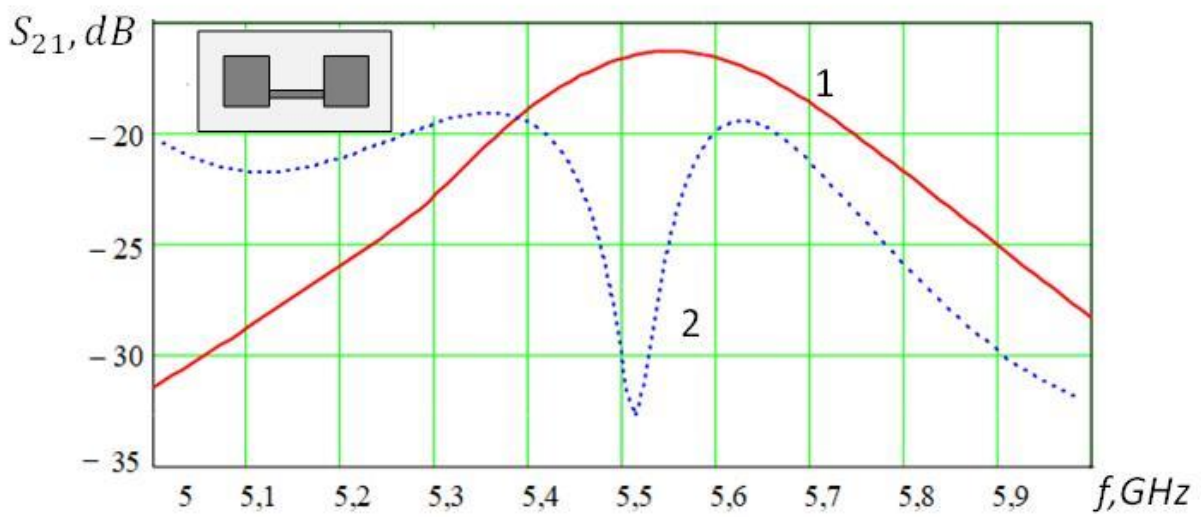

Fig. 7. Antenna with galvanic coupling between patches: 1 - reference antenna; 2 - antenna with transmission line.

This device application increased coupling reduction more than $16 \mathrm{~dB}$, but in narrower frequncy band.

\section{Conclusions}

There were simulated a number of rather simple constructions for mutual coupling suppressing between two microstrip antennas. Their application cause decreasing of correlation coefficient in MIMO antennas. Consequently losses in spectral efficiency [25] decrease too. For example for antenna (fig. 5, c) computer simulation results up to 4.5 times spectral efficiency losses decreasing.

More productive results could be achieved by using numerical optimization methods for choosing elements dimensions and by combination different method [17] of mutual coupling reduction.

\section{References}

1. H. Arun, A.K. Sarma, M. Kanagasabai, S. Velan, C. Raviteja, M.G.N. Alsath, Deployment of modified serpentine structures for mutual coupling reduction in MIMO antennas, IEEE Antennas and wireless propagation letters, 13 (2014) 
2. J. Yang, F. Yang, Z.M. Wang, Reducing mutual coupling of closely spaced microstrip MIMO antennas for WLAN application, IEEE Antennas and Wireless Propagation Letters., 10, 310-313 (2011)

3. R.J. Vaughan, J.B. Andersen, Antenna diversity in mobile communications, Proceedings of the IEEE Transactions on Vehicular Technology, 36, 147-172 (1987)

4. H. Inanoglu, Multiple-input muliple-output system capacity: antennas and propagation aspects, Antennas and Propagation Magazine, 55, 254-273 (2013)

5. M.S. Sharawi, Current misuses and future prospects for printed multiple-input, multiple-output antenna systems, IEEE Antennas and Propagation Magazine, 59, (2017)

6. F. Yang, Y. Rahmat-Samii,. Microstrip antennas integrated with electromagnetic band-gap (EBG) structures: a low mutual coupling design for array applications // IEEE Transactions on Antennas and Propagation, 51, 2936-2946 (2003)

7. N. Ma, H. Zhao, Reduction of the mutual coupling between aperture coupled microstrip patch antennas using EBG structure, IEEE International Wireless Symposium (IWS 2014), 1-4 (2014)

8. A. Habashil, J. Nourinia, C. Ghobadi,. A rectangular defected ground structure (DGS) for reduction of mutual coupling between closely-spaced microstrip antennas, 20th Iranian Conferance on Electrical Engineering, (ICEE2012), May 15-17. Tehran, Iran, (2012)

9. C.Y. Chiu, C.-H. Cheng, R.D. Murch, C.R. Rowell, Reduction of mutual coupling between closely-packed antenna elements. IEEE Transactions on Antennas and Propagation, 55, 1732-1738 (2007)

10. A. Diallo, C. Luxey, P. Le Thuc, R. Staraj, G. Kossiavas. Enhanced two-antenna structures for universal mobile telecommunications system diversity terminals, IET Microwaves, Antennas \& Propagation 2, 94-101 (2008)

11. C.-D. Xue, X.Y. Zhang, Y.F. Cao, Z. Hou, C.F. Ding, MIMO antenna using hybrid electric and magnetic coupling for isolation enhancement DOI 110.1109 /

TAP.2017.2738033, 65, 5162 - 5170 (2017)

12. S. Farsi, H. Aliakbarian, D. Schreurs, B. Nauwelaers, G.A.E. Vandenbosch, Mutual coupling reduction between planar antennas by using a simple microstrip U-section, IEEE Antennas and Wireless Propagation Letters, 11, 1501-1503 (2012)

13. S. Blanch, R. Romeu, I. Corbella, Exact representation of antenna system diversity performance from input parameters description, Electron Lett., 39, 705-707 (2003)

14. J. Li, J.-B. Zhao, J.-J. Liang, L L. Zhong, J. Song, Metamaterial-based planar compact antenna with low mutual coupling, Microwave Journal, (2018)

15. B. Feng, L. Li, J.-C. Cheng, A dual-band dual-polarized stacked microstrip antenna with high-isolation and band-notch characteristics for $5 G$ microcell communications, IEEE Transactions on antennas and propagation, 67, 7 ( 2019)

16. M.F.A. Kadir, M.Z.A.A. Aziz, M.K. Suaidi, M.R. Ahmad, Z. Daud, , M.K.A. Rahim, MIMO beamforming network having polarization diversity, DOI: $10.5772 / 14186$, In book: MIMO Systems, Theory and Applications (2011)

17. M. L. Pablo-González, M. Sánchez-Fernández, E. Rajo-Iglesias, Combination of the three types of diversity to design high-capacity compact MIMO terminals, IEEE Antennas and Wireless Propagation Letters, DOI: 10.1109/LAWP.2014.2336174, 13, 1309-1312 (2014)

18. R.C. Hansen, Phased array antennas, (John Willey \& Sons, 2009) 
19. T. Jiao1, T. Jiang1, Y. Li1, A low mutual coupling MIMO antenna using 3-D electromagnetic isolation wall structures, 2017 Sixth Asia-Pacific Conference on Antennas and Propagation (APCAP), Xian, China, 16-19 Oct. (2017)

20. A. Habashil, J. Nourinia, C. Ghobadi, A rectangular defected ground structure (DGS) for reduction of mutual coupling between closely-spaced microstrip antennas, 20th Iranian Conferance on Electrical Engineering, (ICEE2012), Tehran, Iran, May 15-17, 2012, P. 1347-1351 (2012)

21. O.M. Haraz, A.M. Elboushi, A.-R. Sebak, High-gain slotted oversize patch antenna with electromagnetic bandgap structure, 30th National radio science conference (NRSC 2013), April 16-18 68-73, (2013)

22. K.P. Kumar, H. Khan, Effect of EBG structures on the field pattern of patch antennas, International journal of electromagnetics (IJEL), 1, 1, 13-19 (2016)

23. A. Diallo, C. Luxey, P.L. Thuc, R. Staraj, G. Kossiavas, Study and reduction of the mutual coupling between two mobile phone PIFAs operating in the DCS1800 and UMTS bands, IEEE Transactions on antennas and propagation, 54, 11, 3063-3074 (2006)

24. A. Diallo, C. Luxey, P.L. Thuc, R. Staraj, G. Kossiavas, Enhanced two-antenna structures for universal mobile telecommunications system diversity terminals, IET Microw. Antennas Propag., 2, 1, 94-101 (2008)

25. M. Sharawi, A.Hassan, M. Khan, Correlation coefficient calculations for MIMO antenna systems: a comparative study, International Journal of Microwave and Wireless Technologies, 9, 10, 1991-2004 (2017) 